\title{
TCR mechanobiology: torques and tunable structures linked to early $T$ cell signaling
}

\section{Sun Taek Kim ${ }^{1,2}$, Yongdae Shin ${ }^{3}$, Kristine Brazin ${ }^{1,2}$, Robert J. Mallis ${ }^{1,4}$, Zhen-Yu J. Sun ${ }^{4}$, Gerhard Wagner $^{4}$, Matthew J. Lang ${ }^{5}$ and Ellis L. Reinherz ${ }^{1,2 *}$}

\author{
1 Laboratory of Immunobiology and Department of Medical Oncology, Dana-Farber Cancer Institute, Harvard Medical School, Boston, MA, USA \\ 2 Department of Medicine, Harvard Medical School, Boston, MA, USA \\ ${ }^{3}$ Department of Mechanical Engineering, Massachusetts Institute of Technology, Cambridge, MA, USA \\ ${ }^{4}$ Department of Biological Chemistry and Molecular Pharmacology, Harvard Medical School, Boston, MA, USA \\ ${ }^{5}$ Department of Chemical and Biomolecular Engineering, Vanderbilt University, Nashville, TN, USA
}

\section{Edited by:}

Oreste Acuto, University of Oxford, UK

\section{Reviewed by:}

Oreste Acuto, University of Oxford, UK

Karsten Sauer, The Scripps Research Institute, USA

\section{*Correspondence:}

Ellis L. Reinherz, Cancer Vaccine Center Dana-Farber Cancer Institute, 77 Avenue Louis Pasteur, HIM 419, Boston, MA 02115, USA.

e-mail: ellis_reinherz@dfci. harvard.edu
Mechanotransduction is a basis for receptor signaling in many biological systems. Recent data based upon optical tweezer experiments suggest that the TCR is an anisotropic mechanosensor, converting mechanical energy into biochemical signals upon specific peptide-MHC complex (pMHC) ligation. Tangential force applied along the pseudo-twofold symmetry axis of the TCR complex post-ligation results in the $\alpha \beta$ heterodimer exerting torque on the CD3 heterodimers as a consequence of molecular movement at the T cellAPC interface. Accompanying TCR quaternary change likely fosters signaling via the lipid bilayer predicated on the magnitude and direction of the TCR-pMHC force. TCR glycans may modulate quaternary change, thereby altering signaling outcome as might the redox state of the $\mathrm{CxxC}$ motifs located proximal to the TM segments in the heterodimeric CD3 subunits. Predicted alterations in TCR TM segments and surrounding lipid will convert ectodomain ligation into the earliest intracellular signaling events.

Keywords: quaternary change, mechanosensor, $T$ cell signaling, force transduction, antigen recognition

\section{THE TCR STRUCTURE: OVERVIEW}

The $\alpha \beta$ TCR is a multimeric transmembrane complex composed of a disulfide-linked antigen binding clonotypic heterodimer in non-covalent association with the signal-transducing CD3 subunits (CD3ع, $\mathrm{CD} 3 \varepsilon \delta$, and $\mathrm{CD} 3 \zeta \zeta$ ) (reviewed in Rudolph et al., 2006; Smith-Garvin et al., 2009). TCR signaling via CD3 dimers evokes $\mathrm{T}$ cell lineage commitment and repertoire selection during development, maintains the peripheral $\mathrm{T}$ cell pool, and further differentiates naïve $\mathrm{T}$ cells into effector or memory cell populations upon immune stimulation. Each $\mathrm{CD} 3 \varepsilon, \gamma$, and $\delta$ subunit contains an extracellular immunoglobulin (Ig)-like domain, a membraneproximal stalk region, a transmembrane segment, and a cytoplasmic tail. The interaction between an $\alpha \beta$ TCR heterodimer on the T cell and a pMHC ligand on an antigen-presenting cell (APC) initiates a cascade of downstream signaling events. These events are transmitted via the immunoreceptor tyrosine-based activation motif (ITAM) elements in the cytoplasmic tails of the associated CD3 subunits, whose lengths are substantial relative to those of the TCR $\alpha$ and $\beta$ tails (Reth, 1989; Letourneur and Klausner, 1992; Acuto et al., 2008; van der Merwe and Dushek, 2011) The various CD3 chains induce distinct patterns of cellular protein tyrosine phosphorylation upon activation to recruit intracellular adaptors and signaling molecules. Early, intermediate, and late gene activation programs ensue (Crabtree and Clipstone, 1994). Reviews such as Rudolph et al. (2006) have focused on the structural nature of immune recognition involving $\mathrm{V} \alpha$ and $\mathrm{V} \beta$ domains of a given TCR and its $\mathrm{pMHC}$ ligand. How recognition of pMHC by a weakly interacting $\alpha \beta$ TCR heterodimer on the $\mathrm{T}$ cell surface evokes intracellular signaling via the adjacent $\mathrm{CD} 3$ components of the TCR complex has remained undefined.

Functional TCR $\alpha \beta$ heterodimers were first identified by mAbs on antigen-specific $\mathrm{T}$ cell clones and then $\mathrm{T}$ cell hybridomas (Acuto et al., 1983; Meuer et al., 1983a,b; White et al., 1983). Subsequent sequence analysis of TCRs predicted that they would share with antibodies a common structural basis of ligand recognition, akin to an antibody Fab fragment (Novotny et al., 1986; Chothia et al., 1988). These results agreed with peptide mapping studies of $\alpha$ and $\beta$ subunits which identified conserved as well as variable peptides, implying the existence of constant and variable domains in the TCR $\alpha$ and $\beta$ subunits. The biochemical results were later confirmed and extended by DNA cloning (Chien et al., 1984; Yanagi et al., 1984), and elegantly delineated further by the crystal structure of an intact murine $\alpha \beta$ TCR (Garcia et al., 1996) and a complex between a human TCR, viral peptide, and human MHCI molecule that followed (Garboczi et al., 1996). Structures of TCR $\alpha \beta$ heterodimers and antibody Fab fragments seem very similar. While each of the four TCR $\alpha$ and $\beta$ domains, like those of Fab, has been assigned an Ig fold, deviations are notable in both TCR constant domains (Bentley et al., 1995; Garcia et al., 1996) as well as in the $\mathrm{V} \alpha$ domain (Fields et al., 1995). These deviations define fundamental differences between the TCR as a cell surface receptor and antibody as a soluble immune molecule.

\section{THE C $\beta$ FG LOOP}

First noted upon structural analysis was the striking elongation of the FG loop of the $\mathrm{C} \beta$ domain connecting its $\mathrm{F}$ and $\mathrm{G} \beta$-strands. Compared to other Ig-like structures, there is a 13 amino acid (aa) 
insertion within the FG loop (Kabat et al., 1991). Figure 1A shows a side view of the crystallographically resolved murine N15 MHCIrestricted $\alpha \beta$ TCR (Wang et al., 1998). The unique protrusion of the C $\beta$ domain's FG loop is apparent (boxed). Despite its length, the FG loop is internally well-structured, and constitutes an integrated component of a rigid structural entity that connects the $\mathrm{V} \beta$ and C $\beta$ domains (Wang et al., 1998). This feature of the FG loop is conserved in $\alpha \beta$ TCRs among all mammalian species studied to date, having co-evolved with the development of distinct CD3 $\gamma$ and CD3 $\delta$ genes (Kim et al., 2010). Consistent with the importance implied by this conservation, removal of the FG loop $(\Delta \mathrm{FG})$ in $\mathrm{T}$ cell transfectants as well as TCRs displayed on naïve T cells by transgenesis (Sasada et al., 2002; Touma et al., 2007) affects T cell activation and development. $C \beta \Delta$ FG-expressing T cells manifest reduced proliferation and cytokine production abilities upon $\mathrm{T}$ cell stimulation. Impaired negative selection resulting from the $\mathrm{C} \beta$ FG loop deletion increases the CD $4+\mathrm{CD} 8+$ double positive thymocyte population and causes maturing thymocytes to exit the thymus in larger numbers than their wild-type TCR counterpart.

Second, the overall shape of the TCR C domain module was observed to be remarkably asymmetric. The $\mathrm{C} \beta$ domain is about $55 \AA$ in overall length while that of the $\mathrm{C} \alpha$ is only $40 \AA$. The $\mathrm{C} \beta$ domain bends more acutely toward the $\mathrm{V} \beta$ domain compared to the angle formed between the $\mathrm{C} \alpha$ and $\mathrm{V} \alpha$ domains. About half of the $\mathrm{C} \beta$ domain's ABED $\beta$-sheet is therefore surface exposed, and does not contact the $\mathrm{C} \alpha$ domain. This asymmetry creates a cave-like structure or cavity underneath the $\beta$ chain as shown in the right lower corner of Figure 1A. This cavity measures $\sim 25 \AA$ in depth, $20 \AA$ in height, and $25 \AA$ in width. The partially exposed ABED $\beta$-sheet of the $C \beta$ domain forms an extensive ceiling. The $\mathrm{CD}$ loop and EF loop of the $\mathrm{C} \alpha$ domain (not visualized in this projection) as well as the glycans attached to $\mathrm{C} \alpha \mathrm{N} 185, \mathrm{C} \beta \mathrm{N} 121$, and $\mathrm{C} \beta \mathrm{N} 186$ form one side-wall while the FG loop of $\mathrm{C} \beta$ and the glycan emanating from $\mathrm{C} \beta \mathrm{N} 236$ form a canopy and the other sidewall of the cavity. The glycans project outward and hence, will not occlude the cavity. The $\mathrm{AB}$ loop of the $\mathrm{C} \alpha$ domain, in contrast, projects into this cave (Figure 1A). The floor of the cave is presumably formed by the plasma membrane at the T cell surface. It is noteworthy that the interchain disulfide bond between $\mathrm{C} \alpha$ Cys 213 and C $\beta$ Cys 247 is positioned below the $\mathrm{C} \alpha$ domain, leaving the cavity unobstructed as the TCR $\alpha \beta$ heterodimer projects from the T cell surface membrane. As indicated below, this asymmetric cavity allows for physical and functional linkage of the $\alpha \beta$ heterodimer with $\mathrm{CD} 3 \varepsilon \gamma$ ectodomains. Third, unlike Fabs, the $\alpha \beta$ TCR heterodimers are heavily N-linked glycosylated (Figure 1B). Such adducts can dynamically modify TCR function as noted below. The uniquely kinked conformation of the CD3 $\gamma$ G-strand offers a geometry to accommodate juxtaposition of $\operatorname{CD} 3 \gamma$ and TCR $\beta$ ectodomains and foster quaternary change (see Glossary) that cannot be replaced by the isologous CD $3 \delta$ subunit's extracellular region (Kim et al., 2010).

\section{A DENSE N-LINKED GLYCAN ARRAY DECORATES THE TCR}

The NMR and X-ray structures of CD $3 \varepsilon \gamma$ and $C D 3 \varepsilon \delta$ revealed a unique side-to-side hydrophobic interface with conjoined $\beta$ sheets between the two C/C2-set Ig-like ectodomains. The parallel pairing of these rigidified dimer modules is a striking structural feature not observed elsewhere (Sun et al., 2001, 2004; Arnett et al., 2004; Kjer-Nielsen et al., 2004). Whereas the CD3E subunit conformation is virtually identical in CD $3 \varepsilon \gamma$ and $\mathrm{CD} 3 \varepsilon \delta$, the CD3 $\delta$ ectodomain adopts a C-set Ig fold with a narrower GFC front face $\beta$-sheet that is more parallel to the ABED back face than those $\beta$ sheets in $\mathrm{CD} 3 \varepsilon$ and $\mathrm{CD} 3 \gamma$. The dimer interface between $\mathrm{CD} 3 \delta$ and $\mathrm{CD} 3 \varepsilon$ is highly conserved among species and of similar character as in $\mathrm{CD} 3 \varepsilon \gamma$. Glycosylation sites in $\mathrm{CD} 3 \delta$ are arranged such that the glycans may point away from the membrane, consistent with a model of TCR assembly that allows the CD3 $\delta$ chain to be in close contact with the TCR $\alpha$ chain (Figure 1B). The rigidified CD3 heterodimers are associated with the TCR $\alpha \beta$ heterodimer whose own rigid structure is reinforced by the FG loop and peculiar $\alpha \beta$ asymmetry in constant domains. Thus, not unexpectedly, comparison of unligated and pMHC ligated TCR $\alpha \beta$ structures does not show major conformational changes (reviewed in Rudolph et al., 2006).

A model of the TCR complex of $\alpha \beta, \operatorname{CD} 3 \varepsilon \gamma$, and CD $3 \varepsilon \delta$ ectodomains (Sun et al., 2004) defines a plausible topology and emphasizes its glycan richness (Figure 1B). Given that $\mathrm{CD} 3 \zeta$ has only a nine amino acid long ectosegment, its extracellular segment is omitted from the Figure as are the connecting peptides (CP) of the TCR $\alpha$ and $\beta$ chains and the stalk regions of CD $3 \varepsilon, C D 3 \gamma$, and $\mathrm{CD} 3 \delta$. This rendering incorporates the consequences of several known TCR characteristics: (i) putative transmembrane charge pairs involving TCR subunit chain association with CD3ع-CD $3 \delta-$ $\mathrm{TCR} \alpha-\mathrm{CD} 3 \zeta-\mathrm{CD} 3 \zeta$ as one cluster and CD $3 \varepsilon-\mathrm{CD} 3 \gamma-\mathrm{TCR} \beta$ as a second cluster (Call et al., 2002, 2004), (ii) extracellular domain associations involving other in vitro chain association data (Manolios et al., 1991, 1994), TCR crosslinking results (Brenner, 1985; Koning et al., 1990), and (iii) proximity of one CD3 $\varepsilon$ subunit to the TCR C $\beta$ FG loop revealed by quantitative T cell surface immunofluorescent antibody binding analysis (Ghendler et al., 1998). In addition, structural insights from crystallographic data on the glycosylated N15 TCR $\alpha \beta$ heterodimer ectodomain in complex with $\mathrm{H} 57 \mathrm{Fab}$ and the likely position of glycans in both CD $3 \varepsilon \gamma$ and CD3 $\varepsilon \delta$ (Wang et al., 1998) are considered. Specifically, CD $3 \varepsilon \gamma$ is presumed to be near the cavity formed between the TCR C $\alpha$ CD, EF loops, and the C $\beta$ FG loop (Ghendler et al., 1998; Wang et al., 1998). Residues in the TCR C $\alpha$ AB loop which shows significant conformational change for a LC13 TCR upon pMHC binding (Kjer-Nielsen et al., 2003) were used as target sites for CD3ع $\gamma$ docking in the initial search for possible docking models. $\mathrm{CD} 3 \varepsilon \delta$ is docked on the opposite site of the TCR $\alpha \beta$ domain where there are less glycans to interfere with the more heavily glycosylated CD $3 \delta$ subunit (Figure 1B), and consistent with known TCR $\alpha$ and CD3 $\delta$ TM associations from biochemical analysis (Call et al., 2002).

The multiple N-linked glycan adducts of the TCR complex (Figure 1B, top panel) help guide pMHC ligands to the TCR recognition surface, reducing entropic penalties by directing binding to the exposed, glycan-free CDR loops. Glycans may also serve a regulatory function, contributing to a galectin-glycoprotein lattice (Demetriou et al., 2001). The more heavily glycosylated CD3 $\delta$ subunit may influence TCR subunit assembly through steric constraints. The distribution of glycans in the model shown in Figure 1B is also consistent with the lack of mAbs elicited against the native $\mathrm{CD} 3 \delta$ and $\mathrm{CD} 3 \gamma$ subunits. Importantly, glycans are large and dynamic. These adducts can affect movement of TCR 

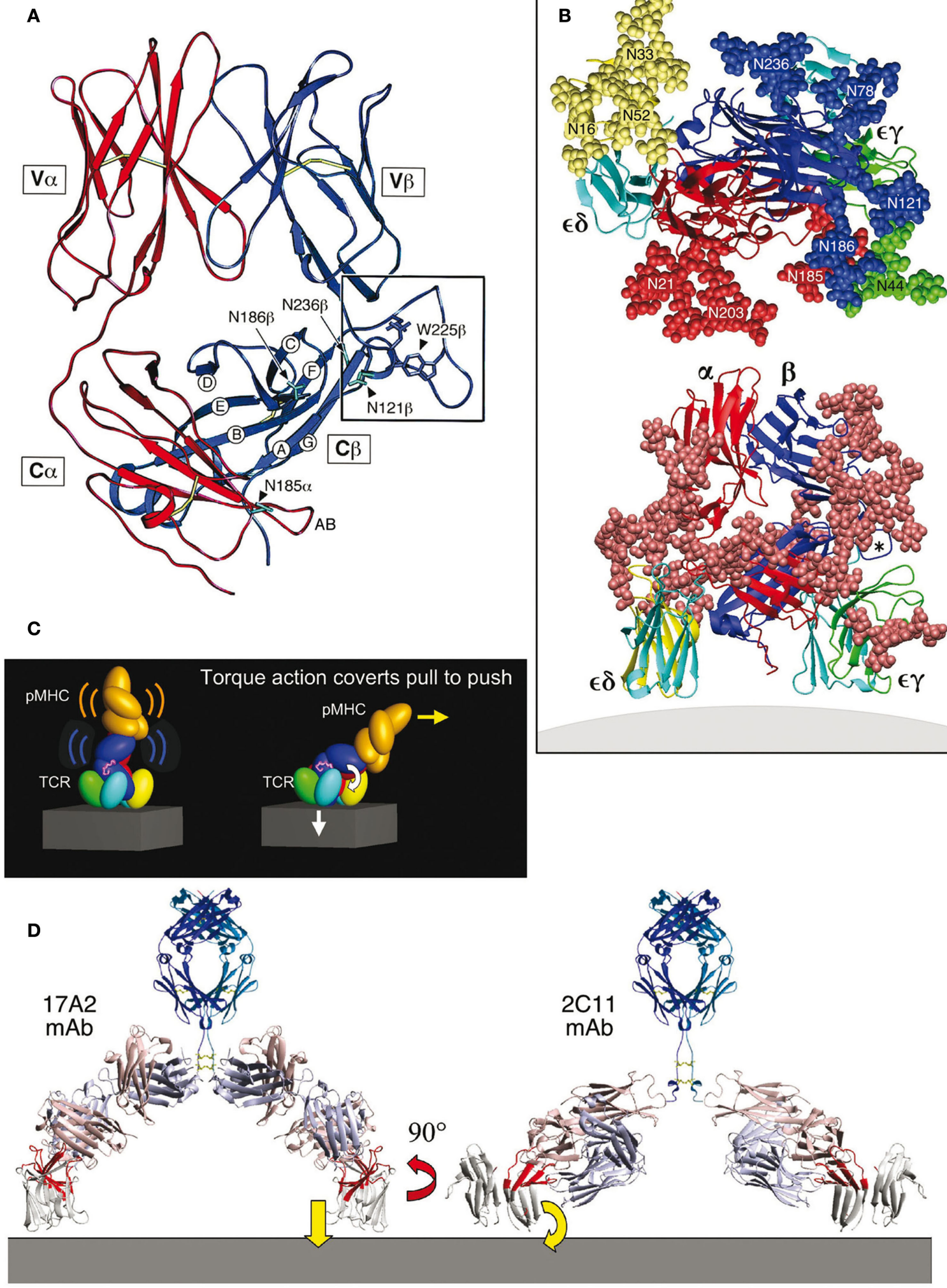

FIGURE 1 | Continued. 


\begin{abstract}
FIGURE 1 | Continued
TCR complex and mechanosensor function model (based on PDB

codes: 1NFD, 1XMW, and 1JBJ). (A) Structure of the TCR $\alpha \beta$ heterodimer ectodomains with the $\alpha$ chain in red and $\beta$ chain in blue. In this side view, the T cell plasma membrane lies at the bottom of the panel [see also lower panel in (B)]. The $\mathrm{C} \beta$-strands are labeled as are N-linked glycosylation sites in the vicinity of the $C \beta$ cave. The $C \beta$ FG loop is boxed and the key hydrophobic residue W225 $\beta$ indicated. The $\mathrm{C} \alpha \mathrm{AB}$ loop (red near N185 $\alpha$ in this projection) points into the cave. (B) The $\alpha \beta$ TCR complex. As shown in the lower panel, the central $\alpha \beta$ heterodimer and surrounding $C D 3 \varepsilon \delta$ and $\mathrm{CD} 3 \varepsilon \gamma$ subunits are left and right, respectively. $C D 3 \varepsilon$ is in light blue, $C D 3 \delta$ is in yellow, and CD3 $\gamma$ is in green. CPK representation (convention for distinguishing atoms of different chemical elements in molecular models) of all glycans is indicated by the rust color spheres. The view is from the
\end{abstract}

side with the T cell membrane at the bottom. In the top panel, a model of TCR complex glycans surrounding the $\mathrm{PMHC}$ binding site from the $\mathrm{pMHC}$ perspective is shown. Individual glycans in CPK representation are numbered and subunit origin color-coded. The C $\beta$ FG loop is denoted with an asterisk. (C) Mechanosensor function of the TCR complex. The left panel shows the pMHC (orange) bound to a TCR complex. As the T cell continues to move prior to stopping, a pull by $\mathrm{pMHC}$ is converted to a push onto $\mathrm{CD} 3 \varepsilon \gamma$ amplified by the $\mathrm{C} \beta \mathrm{FG}$ loop (magenta loop) above CD3 $\varepsilon$ (blue) and accompanying signaling events follow (right panel). (D) Anti-CD3 $\mathrm{mAb}$ binding. The right panel shows that the activating $2 \mathrm{C} 11$ anti-CD3 $\varepsilon$ $m A b$ binds to the exposed outer lobe of $C D 3 \varepsilon$ (highlighted in red) while the non-activating 17A2 anti-CD3 mAb binds perpendicular to the membrane between $\mathrm{CD} 3 \varepsilon$ and $\mathrm{CD} 3 \gamma$ ectodomain subunits (highlighted in red). Anti-CD3 Fab arms are in light blue and salmon colors. subunits, thereby impacting signaling. Consistent with this notion, TCR functional avidity was altered by removal of the $\mathrm{C} \alpha$ glycan (Kuball et al., 2009).

Immediately evident in Figure 1B (bottom panel) is the central position of the TCR $\alpha \beta$ heterodimer with a vertical dimension of $80 \AA$ projecting from the cell membrane, flanked on either side by the shorter $(40 \AA)$ CD3 heterodimers, $\mathrm{CD} 3 \varepsilon \delta$ on the "left" TCR $\alpha$ side and CD3E $\gamma$ on the "right" TCR $\beta$ side. Note that the width of the $C D 3 \varepsilon \delta$ and $C D 3 \varepsilon \gamma$ components, 50 and $55 \AA$, respectively, are comparable in size to that of the TCR $\alpha \beta$ heterodimer $(58 \AA)$, and together (excluding glycans) span $\sim 160 \AA$. These flanking $\mathrm{CD} 3$ ectodomain components will likely impede lateral movement of the TCR $\alpha \beta$ heterodimer upon pMHC binding. As previously noted for CD3ع $\gamma$ (Sun et al., 2001), the intradomain disulfide bridge between Cys residues on the $\mathrm{B}$ and $\mathrm{F}$ strands at the center of each $C D 3 \varepsilon \delta$ domain reinforces the domain structure. Further rigidity for potential signal transduction comes from the paired $\mathrm{G} \beta$-strands in each CD3 heterodimer, coupled with the conserved RxCxxCxE cysteine-coordinated stalks (Sasada et al., 2002) discussed in a separate section.

\section{DYNAMIC QUATERNARY CHANGE UPON TCR LIGATION}

The length of the CD3 subunit stalks (5-10 amino acids) is typical for transmembrane proteins observed, for example, for CD2, CD4, and CD58. On the other hand, the CP found in TCR $\alpha$ (25-26 aa) and TCR $\beta$ (19 aa) are long. The latter are probably mandated by a requirement for a linker segment of sufficient length to span the $50 \AA$ from the end of the interchain disulfide of the TCR $\alpha$ constant domain to the associated $\mathrm{CD} 3 \varepsilon$ and $\mathrm{CD} 3 \delta$ transmembrane (TM) segments which are juxtaposed for apparent charge pairing (i.e., between the TCR $\alpha$ lysine and aspartic residues of $C D 3 \varepsilon$ and CD3 8 TM, respectively). Similar considerations must be applied to the TCR $\beta$ connecting peptide, with charged pairing of the TM TCR $\beta$ lysine with an aspartic and a glutamic acid residue of $\mathrm{CD} 3 \varepsilon$ and CD $3 \gamma \mathrm{TM}$, respectively. Note that the TCR $\alpha \mathrm{TM}$ also includes an arginine residue that is thought to form a charged pair with an aspartic residue in each of the CD3 $\zeta$ TM segments (Call et al., 2002).

We hypothesize that, based on the structures of $C D 3 \varepsilon \delta$ and $C D 3 \varepsilon \gamma$, the highly selective TCR signaling may require dynamic interaction rather than static on-and-off switching, such that the interfaces between the extracellular domains of the TCR $\alpha \beta$ heterodimer and CD3 dimers may be quite small. With this current model, no detailed information on the interfaces is warranted, being one of a range of acceptable structures. Nonetheless, we envisage the ectodomains of TCR $\alpha \beta$ chains being supported by the $\mathrm{CD} 3$ heterodimers, while components of the TCR $\alpha \beta$ dimer, such as the C $\beta$ FG loop (Wang et al., 1998) and the $\alpha$-CP (Backström et al., 1998; Werlen et al., 2000) may serve as levers and/or tension elements to help control vertical movements of CD3 subunits for signal transduction through the critical TM segments. Given apparently weak ectodomain association between $\mathrm{CD} 3$ and TCR $\alpha \beta$ heterodimers (see Arnett et al., 2004; Touma et al., 2007 and references therein), it is likely that this assembly undergoes dynamic quaternary change upon TCR ligation and triggering, thereby affecting cytoplasmic $\mathrm{CD} 3$ signaling regions. According to the model, the five helices of the $\mathrm{CD} 3 \varepsilon-\mathrm{CD} 3 \delta-\mathrm{TCR} \alpha-\mathrm{CD} 3 \zeta-\mathrm{CD} 3 \zeta$ component lie closer to the TCR $\alpha$ subunit and the three helices of the $\mathrm{CD} 3 \varepsilon-\mathrm{CD} 3 \gamma-\mathrm{TCR} \beta$ component lie closer to the TCR $\beta$ subunit (Call et al., 2002).

\section{THE TCR AS AN ANISOTROPIC MECHANOSENSOR}

The squat and rigid CD3 connecting segments (Touma et al., 2007) contrast sharply with the long and flexible TCR $\alpha$ and $\beta$ CP linking their respective constant domains to the transmembrane segments. Structural insight into a basis for this contrasting arrangement first came from analysis of interactions of activating and non-activating anti-CD3\& monoclonal antibodies, which bind to

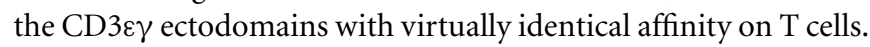
Activating antibodies footprint to the membrane distal $\mathrm{CD} 3 \varepsilon$ lobe which they approach diagonally, adjacent to the lever-like C $\beta$ FG loop noted above to facilitate pMHC-triggered activation. In contrast, a non-activating $\mathrm{mAb}(17 \mathrm{~A} 2)$ was found to bind to the cleft between $\mathrm{CD} 3 \varepsilon$ and $\gamma$ in a perpendicular mode (Kim et al., 2009; Figure 1D). Thus, polystyrene bead-bound 17A2 antibody became stimulatory only upon application of $\sim 50 \mathrm{pN}$ of external tangential force to the bead. Specific bead-bound pMHC (but not irrelevant peptide bound to the same MHC) activates a T cell upon application of a similar force via optical tweezers to initiate intracellular calcium flux (Figure 2). These findings imply that the TCR is a mechanosensor, converting mechanical energy into a biochemical signal upon specific pMHC ligation that occurs as a T cell moves over APCs during the course of immune surveillance. As shown in Figure 1C (left panel), the pMHC on the APC is first ligated by a specific TCR. However, as the T cell continues to move prior to a stop movement signal mediated through insideout integrin affinity up-regulation, pMHC functions as a force transducing handle to pull on the TCR $\alpha \beta$ heterodimer (Figure 1C, 

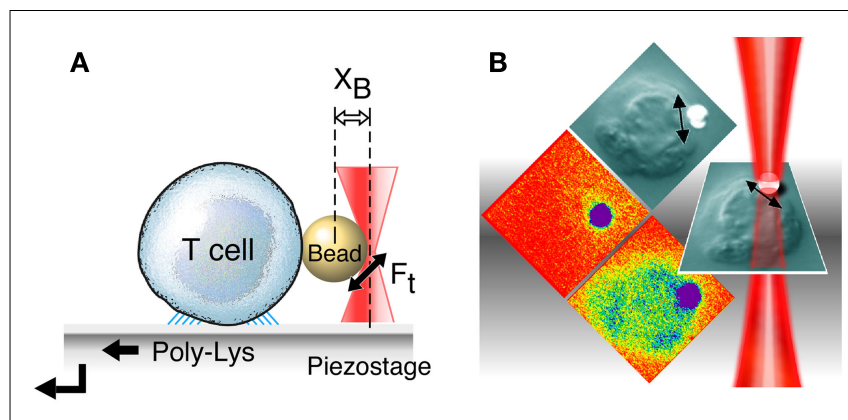

FIGURE 2 |T cell activation using optical tweezers. (A) Mechanical force application using optical tweezers. AT cell is immobilized on poly L-Lysine coated cover glass. After a trapped ligand-coated polystyrene bead is guided into contact with a surface-bound $T$ cell using an automated piezoelectric stage, a continuous sinusoidal tangential force is applied to the bead by moving the trapping laser back and forth $\left(X_{B}\right.$, distance from the trap center to the bead center; Ft, applied force by optical trapping laser). (B) Calcium flux in T cells after application of external mechanical force using optical tweezers. Optical tweezers are employed for application of force (double headed arrow) to a polystyrene bead (white) arrayed with specific $T$ cell receptor ligand ( $\mathrm{pMHC}$, in this case $\mathrm{VSV} 8 / \mathrm{K}^{\mathrm{b}}$ ). The bead abutting the surface of a single T cell (naïve N15 TCR transgenic T lymphocyte) is shown in the DIC image as gray. The tangential force $(50 \mathrm{pN})$ along the T cell surface triggers a rise in intracellular calcium flux shown in colorization through an increase in green intensity (bottom panel). Without force application, the bound ligand induces no increase in calcium (middle panel).

right panel). This force is amplified and exerted on CD3e $\gamma$ by the lever arm where the TCR $\beta$ TM acts as a fulcrum. For activation, force must be applied to the TCR complex tangentially and not perpendicular to the plane of the $\mathrm{T}$ cell membrane, showing that the TCR is an anisotropic mechanosensor (i.e., direction matters; Kim et al., 2009). The rupture force and bond lifetime under load between pMHC and TCR $\alpha \beta$ heterodimer are potentially important parameters which can determine the potency of pMHC stimulation. The pull from pMHC most probably causes the $\mathrm{C} \beta$ FG loop to push on the upper outer lobe of CD3e. During this force driven quaternary change, TCR-decorating glycans can serve as steric and spring-like barriers that require force to overcome in order to deliver signaling to CD3 subunits. Several groups have recently provided evidence that physical force applied to TCR components activates T cells (Kim et al., 2009; Li et al., 2010; Ma and Finkel, 2010; Husson et al., 2011; Judokusumo et al., 2012).

That the TCR is a mechanosensor activated by directionspecific physical force has several immediate implications. First, since the total force applied to the $\mathrm{T}$ cell surface is essentially defined during movement of the T cell relative to that of the APC, ligation of a small number of TCRs by several cognate pMHCs on the opposing APC will exert a greater physical force on each individual TCR than ligations of multiple TCRs on the same T cell by a large number of pMHCs on the APC. Hence, specificity and sensitivity are built into TCR mechanosensor function. Second, in principle, shear forces generated by $\mathrm{T}$ cell movement during immune surveillance can form catch bonds at the TCR-pMHC interface to enhance binding and/or confer additional ligand specificity. These bonds, which are strengthened by tensile force, have been described for cell adhesion molecules (Marshall et al., 2003). Catch bond characteristics may allow for non-linear response in the lifetime of TCR-pMHC bonding where it is maximal at certain force levels enabling specific pMHC ligand to drive quaternary change, yet allow for quick release for other pMHC ligand binding. Because the torque exerted by the TCR-pMHC interaction around the C $\beta$ FG loop/CD3 $\varepsilon \gamma$ "flywheel" is dependent on the force applied, the length of the $\alpha \beta-$ pMHC lever arm and the angle between the force vector and lever arm, TCR docking topology is important.

Consistent with this view, a recent paper demonstrated that docking orientation rather than affinity of $3 \mathrm{D}$ binding correlated with the ligand's T cell activating potential (Adams et al., 2011). This finding is also in agreement with a docking orientation difference between the $\mathrm{T}$ cell activating $2 \mathrm{C} 11$ versus non-activating 17A2 antibodies (Kim et al., 2009; Figure 1D). Bacterial superantigens stimulate up to $20 \%$ of the entire T cell population by simultaneously interacting with class II MHC and TCR $\beta$ molecules on APC and T cells, respectively (Sundberg et al., 2002). Not surprisingly, therefore, these interactions foster TCR docking vectors similar to those of activating TCR-pMHC interactions (Reinherz et al., 1999). Precedent for mechanoreceptors in the hematopoietic system is the von Willebrand factor (VWF) receptor on platelets where tensile stress on bonds between the GPIb $\alpha$ subunit and the VWFA1 domain under fluid dynamic conditions triggers integrin $\alpha_{\mathrm{IIb}} \beta_{\mathrm{III}}$ activation to support platelet adhesion (Doggett et al., 2002; Ruggeri, 2007).

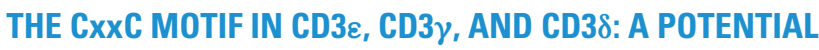 ROLE IN STRUCTURAL STABILIZATION AND REDOX SENSITIVE SIGNALING ATTENUATION}

Studies on murine CD3 $\gamma$ (Touma et al., 2007) and human CD3 $\gamma$ (Thomassen et al., 2006; Xu et al., 2006) attest to the importance of the cysteines in the CxxC motif for TCR function and/or assembly. Similar findings have been shown for CD3e (Martínez-Martín et al., 2009; Wang et al., 2009). Given that the two cysteines in each CD3 CxxC motif are adjacent to the TM helix (Figure 3) and in view of a recent study showing that a $\mathrm{CxxC}$ motif is found at the $\mathrm{N}$-termini of $\alpha$-helices, stabilizing $\alpha$-helical structures, this juxtaposition is noteworthy (Iqbalsyah et al., 2006). Assuming an intrachain disulfide is formed in each stalk region (vide infra), one possibility is that the CD3 TM helix is stabilized and perhaps even extended as an elongated helix above the plane of the cell membrane. Alternatively, this $\mathrm{CxxC}$ motif may support a tight $\beta$ turn (Hsu et al., 2006). In either case, the disposition of the CD3 ectodomain relative to the cell membrane may be affected if a disulfide bond is removed, attenuating signaling, and altering TCR quaternary structure. The disulfide bonds would ensure that lever action on the TM helices of the various CD3 domains would be simultaneous, parallel, and in phase. Whether physiologic modification of the redox state of the CD3 heterodimer is regulated during development or $\mathrm{T}$ cell activation can be determined. However, given that TCR crosslinking on murine and human T lymphocytes generates hydrogen peroxide and superoxide ions (Pani et al., 2000; Devadas et al., 2002) and that oxidative stress from macrophages alters the native $\mathrm{CD} 3 \zeta$ association with the TCR (Otsuji et al., 1996), it is possible that redox reactivity of CD3 stalk cysteines is critical for modulating TCR quaternary structure, subunit conformation, and functional responsiveness. Rapid conversion between oxidized and reduced forms under 


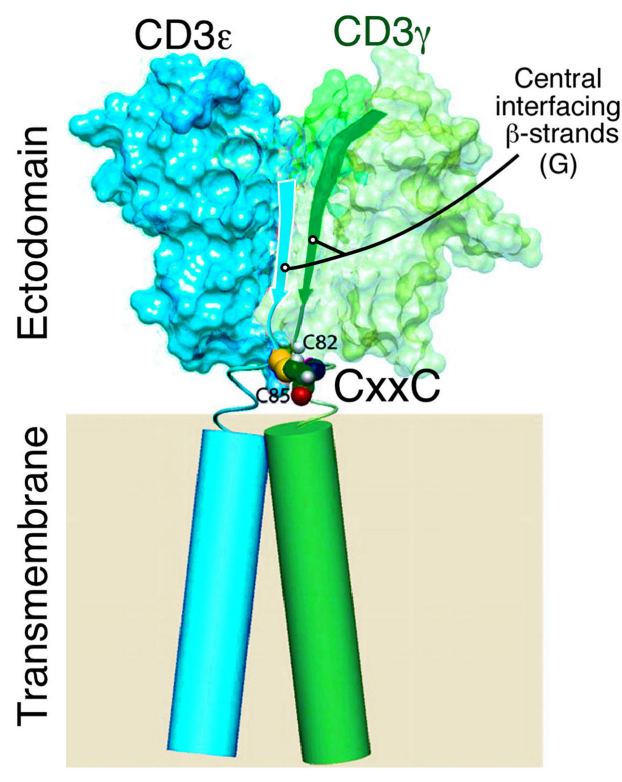

FIGURE 3 | Model of CD3 $\varepsilon \gamma$ heterodimer with CxxC motif proximal to TM segments. Model showing NMR-defined domains of $C D 3 \varepsilon \gamma$ highlighting the central $\beta$ strands of the ectodomain, the $\mathrm{Cxx} C$ connecting peptide region (CD3 $\gamma$ C82 and $\mathrm{C} 85$ cysteines only shown for clarity) and the transmembrane domains of unknown structures (cylinders) drawn to scale (Touma et al., 2007).

physiologic circumstances may be important for TCR triggering and downregulation, respectively. Assuming a direct link between redox state, TCR function, and TM structure is demonstrated, future efforts can be directed toward design of deliverable redox regulators using $\mathrm{mAb}$ or other materials to modify $\mathrm{T}$ cell responses.

\section{MECHANOSENSING AT THE IMMUNOLOGICAL SYNAPSE}

Our data involve a model wherein tangential forces applied along the pseudo-twofold symmetry axis of the TCR $\alpha \beta$ heterodimer exerts a highly selective signaling torque on the CD3 components. This directionally specified vector precludes non-specific activation and fosters antigen-specific events. In turn, activation leads to stop movement and formation of the immunological synapse. Mechanoreceptor function is most likely additionally tunable by inducible actin cytoskeletal interactions with TCR and pMHC on $\mathrm{T}$ cells and APCs, respectively, since torque will be greatest in their presence. Within the synapse, force could be generated on the TCR via microcluster formation and actin-based trafficking (Yokosuka et al., 2008). Mechanosensing can be further amplified at the synapse where intermembrane distances $(\sim 150 \AA)$ are optimal for TCR/pMHC ligation in conjunction with other signaling molecules to sustain activation from both p-SMAC and c-SMAC components. The facilitating roles of adhesion molecules and CD4 and CD8 co-receptors have been discussed in detail previously (Kim et al., 2009 and references therein) and will not be reviewed here. Rapid transport of TCRs to the immunological synapse and TCR signaling after disengagement of pMHC likely gives rise to serial TCR triggering for high activation efficiency within the immunological synapse. In this dynamic anisotropic mechanosensor model, signaling occurs via the built-in geometry of the TCR-pMHC complex and there is no requirement for TCR or pMHC oligomerization via "permissive" geometry on the T cell membrane. Soluble anti-TCR antibody or pMHC oligomers activate by mediating force in an artificial mode, such as through chemical forces resulting from their specific binding geometries, but nevertheless dependent on mechanosensor function.

\section{FUTURE DIRECTIONS}

Details of the mechanobiology of TCR function remain to be fully elucidated. Force threshold requirements and effects on signaling of loading rate and directionality of pMHC ligand movement relative to the TCR need to be established. Function/structure studies of alterations attenuating TCR rigidity with respect to $\mathrm{T}$ cell activation are needed.

The precise mechanotransduction of TCR signaling upon modification of the redox state of the CD3 heterodimer, for example, can be measured using a combined fluorescence and optical trap microscope for simultaneous trapping and fluorescence imaging (Tarsa et al., 2007). This newly established methodology offers nanometer level position, piconewton level force, and low light single molecule fluorescence sensitivity. Furthermore, quaternary motion and the mechanical properties of the CD3 heterodimer via single molecule studies on intact $\mathrm{T}$ cells can be compared. Finally, the physical force relayed from the ectodomain onto the TM during mechanosensor function may be transmitted to the cytoplasmic tail directly and/or indirectly by modification of the membrane lipid organization (Zech et al., 2009; Nika et al., 2010). The CD3 $\varepsilon$ cytoplasmic tail maintains close interaction with the plasma membrane via basic CD $3 \varepsilon$ residues and acidic phospholipids enriched in the inner leaflet of the plasma membrane ( $\mathrm{Xu}$ et al., 2008; Deford-Watts et al., 2009). Two key tyrosine residues in the CD $3 \varepsilon$ ITAM are deeply inserted in the hydrophobic core of the lipid bilayer. Release of sequestered tyrosines must occur for phosphorylation by Lck to follow. $\mathrm{CD} 3 \zeta$ phosphorylation is also lipid-dependent (Aivazian and Stern, 2000) and the importance of the six ITAMs in the $\zeta \zeta$ homodimer for signaling is not to be underestimated (Acuto et al., 2008 and references therein).

$\mathrm{T}$ cells are exposed to stresses from fluid and cell motions during immune surveillance. pMHC ligands are tethered on APCs via their own TM segments. pMHC mobility may be tunable via actin cytoskeletal connections during dendritic cell maturation, thereby altering $\mathrm{T}$ cell activation. $\mathrm{T}$ cells may exploit stress and geometrical cues from this greater micro-environment to discern proper signaling from noise, identifying appropriate TCRpMHC interactions. The dynamic quaternary structure of the TCR may not only be controlled through glycosylation and redox modification but through non-linear response to forces through mechanisms like catch bonds, where pushing and pulling on the TCR facilitates mechanisms such as conformational change, allostery and stabilization that the TCR may exploit for robust and proper signaling. Aside from offering basic scientific insight into multisubunit receptor mobility and function, that understanding of early TCR signal initiation will be advantageous for drug development aimed at modifying $\mathrm{T}$ cell activation.

\section{GLOSSARY}

Shear force Force which acts tangentially to a surface. Here, we use "tangential force" interchangeably. 
Tensile force Force which acts perpendicular to a surface, like the force on a rope.

Catch bond Type of intermolecular bonds which strengthens upon application of force. Typically, force accelerates bond breakage as in "slip bonds."

Torque The tendency of force to generate rotation of a body about an axis.

Lever Force amplifying device which consists of a fulcrum, fixed pivot, and a rigid beam.

Chemical force Force derived from intermolecular bonding.

Mechanotransduction Cell signaling through means of a mechanical input.

\section{REFERENCES}

Acuto, O., Di Bartolo, V., and Michel, F. (2008). Tailoring T-cell receptor signals by proximal negative feedback mechanisms. Nat. Rev. Immunol. 8, 699-712.

Acuto, O., Hussey, R. E., Fitzgerald, K. A., Protentis, J. P., Meuer, S. C., Schlossman, S. F., and Reinherz, E. L. (1983). The human $\mathrm{T}$ cell receptor: appearance in ontogeny and biochemical relationship of the $\alpha$ and $\beta$ subunits on IL-2 dependent clones and T cell tumors. Cell 34, 717-726.

Adams, J. J., Narayanan, S., Liu, B., Birnbaum, M. E., Kruse, A., Bowerman, N. A., Chen, W., Levin, A. M., Connolly, J. M., Zhu, C., Kranz, D. M., and Garcia, K. C. (2011). T cell receptor signaling is limited by docking geometry to peptide-major histocompatibility complex. Immunity 35, 681-693.

Aivazian, D., and Stern, L. J. (2000). Phosphorylation of $\mathrm{T}$ cell receptor zeta is regulated by a lipid dependent folding transition. Nat. Struct. Biol. 7, 1023-1026.

Arnett, K. L., Harrison, S. C., and Wiley, D. C. (2004). Crystal structure of a human CD3-epsilon/delta dimer in complex with a UCHT1 single-chain antibody fragment. Proc. Natl. Acad. Sci. U.S.A. 101, 16268-16273.

Backström, B. T., Muller, U., Hausmann, B., and Palmer, E. (1998). Positive selection through a motif in the alphabeta T cell receptor. Science 281, 835-838.

Bentley, G. A., Boulot, G., Karjalainen, K., and Mariuzza, R. A. (1995). Crystal structure of the $\beta$ chain of a $\mathrm{T}$ cell antigen receptor. Science 267, 1984-1987.

Brenner, M. B. (1985). Crosslinking of human $\mathrm{T}$ cell receptor proteins: association between the $\mathrm{T}$ cell idiotype $\beta$ subunit and the T3 glycoprotein heavy subunit. Cell 40, 183-190.
Call, M. E., Pyrdol, J., Wiedmann, M., and Wucherpfennig, K. W. (2002). The organizing principle in the formation of the $\mathrm{T}$ cell receptor-CD3 complex. Cell 111, 967-979.

Call, M. E., Pyrdol, J., and Wucherpfennig, K. W. (2004). Stoichiometry of the T-cell receptor-CD3 complex and key intermediates assembled in the endoplasmic reticulum. EMBOJ. 23, 2348-2357.

Chien, Y., Becker, D. M., Lindsten, T., Okamura, M., Cohen, D. I., and Davis, M. M. (1984). A third type of murine $\mathrm{T}$ cell receptor gene. Nature 312, 31-35.

Chothia, C., Boswell, D. R., and Lesk, A. M. (1988). The outline structure of the $\mathrm{T}$ cell $\alpha \beta$ receptor. EMBO J. 7, 3745-3755.

Crabtree, G. R., and Clipstone, N. A. (1994). Signal transmission between the plasma membrane and nucleus of $\mathrm{T}$ lymphocytes. Annu. Rev. Biochem. 63, 1045-1083.

Deford-Watts, L. M., Tassin, T. C., Becker, A. M., Medeiros, J. J., Albanesi, J. P., Love, P. E., Wülfing, C., and van Oers, N. S. (2009). The cytoplasmic tail of the $\mathrm{T}$ cell receptor CD3 epsilon subunit contains a phospholipid-binding motif that regulates $\mathrm{T}$ cell functions. $J$. Immunol. 183, 1055-1064.

Demetriou, M., Granovsky, M., Quaggin, S., and Dennis, J. M. (2001). Negative regulation of $\mathrm{T}$ cell activation and autoimmunity by Mgat $5 \mathrm{~N}$ glycosylation. Nature 409, 733-739.

Devadas, S., Zaritskaya, L., Rhee, S. G., Oberley, L., and Williams, M. S. (2002). Discrete generation of superoxide and hydrogen peroxide by $\mathrm{T}$ cell receptor stimulation: selective regulation of mitogen-activated protein kinase activation and fas ligand expression. J. Exp. Med. 195, 59-70.

Doggett, T. A., Girdhar, G., Lawshé, A., Schmidtke, D. W., Laurenzi,

Loading rate Effective loading velocity in units of Newtons per second over which force is applied.

Quaternary change Structural reorientation of subunits within a multisubunit protein complex that may regulate physiologic function.

2D binding As membrane-associated molecules are restricted to diffuse in two dimensions, their binding properties are stated in two-dimensional (2D) units, in contrast with solution binding properties, which are three-dimensional (3D).

\section{ACKNOWLEDGMENTS}

We thank Dr. Jia-Huai Wang for thoughtful comments. This work was supported by NIH grant AI19807.

I. J., Diamond, S. L., and Diacovo, T. G. (2002). Selectin-like kinetics and biomechanics promote rapid platelet adhesion in flow: the GPIb(alpha)-vWF tether bond. Biophys. J. 83, 194-205.

Fields, B. A., Ober, B., Malchiodi, E. L., Lebedeva, M. I., Braden, B. C., Ysern, X., Kim, J. K., Shao, X., Ward, E. S. and Mariuzza, R. A. (1995). Crystal structure of the $\mathrm{V} \alpha$ domain of a $\mathrm{T}$ cell antigen receptor. Science 270, 1821-1824.

Garboczi, D. N., Ghosh, P., Utz, U., Fan, Q. R., Biddison, W. E., and Wiley, D. C. (1996). Structure of the complex between human $\mathrm{T}$ cell receptor, viral peptide and HLA-A2. Nature 384, 134-141.

Garcia, K. C., Degano, M., Stanfield, R. L., Brunmark, A., Jackson, M. R., Peterson, P. A., Tayton, L., and Wilson, I. A. (1996). An $\alpha \beta$ T cell receptor structure at $2.5 \AA$ and its orientation in the TCR-MHC complex. Science 274, 209-219.

Ghendler, Y., Smolyar, A., Chang, H. C., and Reinherz, E. L. (1998). One of the CD3epsilon subunits within a $\mathrm{T}$ cell receptor complex lies in close proximity to the Cbeta FG loop. J. Exp. Med. 187, 1529-1536.

Hsu, H. J., Chang, H. J., Peng, H. P. Huang, S. S., Lin, M. Y., and Yang, A. S. (2006). Assessing computational amino acid beta turn propensities with a phage-displayed combinatorial library and directed evolution. Structure 14, 1499-1510.

Husson, J., Chemin, K., Bohineust, A., Hivroz, C., and Henry, N. (2011). Force generation upon $\mathrm{T}$ cell receptor engagement. PLoS ONE 6, e19680. doi:10.1371/journal.pone.0019680

Iqbalsyah, T. M., Moutevelis, E., Warwicker, J., Errington, N., and Doig, A. J. (2006). The CXXC motif at the N terminus of an alpha-helical peptide. Protein Sci. 15, 1945-1950.
Judokusumo, E., Tabdanov, E., Kumari, S., Dustin, M. L., and Kam, L. C. (2012). Mechanosensing in T lymphocyte activation. Biophys. J. 102, L05-L07.

Kabat, E. A., Wu, T. T., Perry, H. M., Gottesman, K. S., and Faeller, C. (1991). Sequences of Proteins of Immunological Interest. Bethesda: U.S. Dept. of Health and Human Services, DHS, NIH.

Kim, S. T., Takeuchi, K., Sun, Z. Y., Touma, M., Castro, C. E., Fahmy, A., Lang, M. J., Wagner, G., and Reinherz, E. L. (2009). The alphabeta $\mathrm{T}$ cell receptor is an anisotropic mechanosensor. J. Biol. Chem. 284, 31028-31037.

Kim, S. T., Touma, M., Takeuchi, K., Sun, Z. Y., Dave, V. P., Kappes, D. J., Wagner, G., and Reinherz, E. L. (2010). Distinctive CD3 heterodimeric ectodomain topologies maximize antigentriggered activation of alpha beta $\mathrm{T}$ cell receptors. J. Immunol. 185 , 2951-2959.

Kjer-Nielsen, L., Clements, C. S., Purcell, A. W., Brooks, A. G., Whisstock, J. C., Burrows, S. R., McCluskey, J., and Rossjohn, J. (2003). A structural basis for the selection of dominant alphabeta $\mathrm{T}$ cell receptors in antiviral immunity. Immunity 18 , 53-64.

Kjer-Nielsen, L., Dunstone, M. A., Kostenko, L., Ely, L. K., Beddoe, T., Mifsud, N. A., Purcell, A. W., Brooks, A. G., McCluskey, J., and Rossjohn, J. (2004). Crystal structure of the human $\mathrm{T}$ cell receptor CD3 epsilon gamma heterodimer complexed to the therapeutic mAb OKT3. Proc. Natl. Acad. Sci. U.S.A. 101, 7675-7680.

Koning, F., Maloy, W. L., and Coligan, J. E. (1990). The implications of subunit interactions for the structure of the T cell receptor-CD3 complex. Eur. J. Immunol. 20, 299-305. 
Kuball, J., Hauptrock, B., Malina, V., Antunes, E., Voss, R. H., Wolfl, M., Strong, R., Theobald, M., and Greenberg, P. D. (2009). Increasing functional avidity of TCR-redirected $\mathrm{T}$ cells by removing defined $\mathrm{N}$ glycosylation sites in the TCR constant domain. J. Exp. Med. 206, 463-475.

Letourneur, F., and Klausner, R. D. (1992). Activation of T cells by a tyrosine kinase activation domain in the cytoplasmic tail of CD3 epsilon. Science 255, 79-82.

Li, Y. C., Chen, B. M., Wu, P. C., Cheng, T. L., Kao, L. S., Tao, M. H., Lieber, A., and Roffler, S. R. (2010). Cutting Edge: mechanical forces acting on T cells immobilized via the TCR complex can trigger TCR signaling. J. Immunol. 184, 5959-5963.

Ma, Z., and Finkel, T. H. (2010). T cell receptor triggering by force. Trends Immunol. 31, 1-6.

Manolios, N., Kemp, O., and Li, Z. G. (1994). The $T$ cell antigen receptor $\alpha$ and $\beta$ chains interact via distinct regions with CD3 chains. Eur. J. Immunol. 24, 84-92.

Manolios, N., Letourneur, F., Bonifacino, J. S., and Klausner, R. D. (1991). Pairwise, cooperative and inhibitory interactions describe the assembly and probable structure of the Tcell antigen receptor. $E M B O \mathrm{~J} .10$, 1643-1651.

Marshall, B. T., Long, M., Piper, J. W., Yago, T., McEver, R. P., and Zhu, C. (2003). Direct observation of catch bonds involving cell-adhesion molecules. Nature 423, 190-193.

Martínez-Martín, N., Risueño, R. M., Morreale, A., Zaldívar, I., FernándezArenas, E., Herranz, F., Ortiz, A. R., and Alarcón, B. (2009). Cooperativity between $\mathrm{T}$ cell receptor complexes revealed by conformational mutants of CD3epsilon. Sci. Signal. 2, ra43.

Meuer, S. C., Acuto, O., Hussey, R. E., Hodgdon, J. C., Fitzgerald, K. A., Schlossman, S. F., and Reinherz, E. L. (1983a). Evidence for the T3associated $90 \mathrm{KD}$ heterodimer as the $\mathrm{T}$ cell antigen receptor. Nature 303, 808-810.

Meuer, S. C., Fitzgerald, K. A., Hussey, R. E., Hodgdon, J. C., Schlossman, S. F., and Reinherz, E. L. (1983b). Clonotypic structures involved in antigen specific human $\mathrm{T}$ cell function: relationship to the T3 molecular complex. J. Exp. Med. 157, 705-719.

Nika, K., Soldani, C., Salek, M., Paster, W., Gray, A., Etzensperger, R., Fugger, L., Polzella, P., Cerundolo, V.,
Dushek, O., Höfer, T., Viola, A., and Acuto, O. (2010). Constitutively active Lck kinase in $\mathrm{T}$ cells drives antigen receptor signal transduction. Immunity 32, 766-777.

Novotny, J., Tonegawa, S., Saito, H., Kranz, D. M., and Eisen, H. N. (1986). Secondary, tertiary, and quaternary structure of T-cell-specific immunoglobulin-like polypeptide chains. Proc. Natl. Acad. Sci. U.S.A. 83, 742-746.

Otsuji, M., Kimura, Y., Aoe, T., Okamoto, Y., and Saito, T. (1996). Oxidative stress by tumor-derived macrophages suppresses the expression of $\mathrm{CD} 3 \zeta$ chain of T-cell receptor complex and antigen-specific $\mathrm{T}$ cell responses. Proc. Natl. Acad. Sci. U.S.A. 93, 13119-13124.

Pani, G., Colavitti, R., Borrello, S., and Galeotti, T. (2000). Endogenous oxygen radicals modulate protein tyrosine phosphorylation and JNK-1 activation in lectin-stimulated thymocytes. Biochem. J. 347, 173-181.

Reinherz, E. L., Tan, K., Tang, L., Kern, P., Liu, J.-H., Xiong, Y., Hussey, R. E., Smolyar, A., Hare, B., Zhang, R., Joachimiak, A., Chang, H. C., Wagner, G., and Wang, J. (1999). The crystal structure of a $\mathrm{T}$ cell receptor in complex with peptide and MHC class II. Science 286, 1913-1921.

Reth, M. (1989). Antigen receptor tail clue. Nature 338, 383-384.

Rudolph, M. G., Stanfield, R. L., and Wilson, I. A. (2006). How TCRs bind MHCs, peptides, and coreceptors. Annu. Rev. Immunol. 24, 419-466.

Ruggeri, Z. M. (2007). Von Willebrand factor: looking back and looking forward. Thromb. Haemost. 98, 55-62.

Sasada, T., Touma, M., Chang, H.-C., Clayton, L. K., Wang, J., and Reinherz, E. L. (2002). Involvement of the TCR C $\beta$ FG loop in thymic selection and T cell function. J. Exp. Med. 195, 1419-1431.

Smith-Garvin, J. E., Koretzky, G. A., and Jordan, M. S. (2009). T cell activation. Annu. Rev. Immunol. 27, 591-619.

Sun, Z. J., Kim, K. S., Wagner, G., and Reinherz, E. L. (2001). Mechanisms contributing to $\mathrm{T}$ cell receptor signaling and assembly revealed by the solution structure of an ectodomain fragment of the $\mathrm{CD} 3$ epsilon gamma heterodimer. Cell 105, 913-923.

Sun, Z.-Y. J., Kim, S. T., Kim, I. L., Fahmy, A., Reinherz, E. L., and Wagner, G. (2004). Solution structure of the $\mathrm{CD} 3 \epsilon \delta$ ectodomain and comparison with $C D 3 \in \gamma$ as a basis for modeling $\mathrm{T}$ cell receptor topology and signaling. Proc. Natl. Acad. Sci. U.S.A. 101, 16867-16872.

Sundberg, E. J., Li, Y. C., and Mariuzza, R. A. (2002). So many ways of getting in the way: diversity in the molecular architecture of superantigendependent $\mathrm{T}$ cell signaling complexes. Curr. Opin. Immunol. 14 36-44.

Tarsa, P. B., Brau, R. R., Barch, M., Ferrer, J. M., Freyzon, Y., Matsudaira, P., and Lang, M. J. (2007). Detecting forceinduced molecular transitions with fluorescence resonant energy transfer. Angew. Chem. Int. Ed. Engl. 46, 1999-2001.

Thomassen, E. A. J., Dekking, E. H. A., Thompson, A., Franken, K. L., O'Sanal, O., Abrahams, J. P., van Tol, M. J. D., and Koning, F. (2006). The impact of single amino acid substitutions in $\mathrm{CD} 3 \gamma$ on the $\mathrm{CD} 3 \epsilon \gamma$ interaction and T-cell receptor-CD3 complex formation. Hum. Immunol. $67,579-588$.

Touma, M., Sun, Z. Y., Clayton, L. K., Marissen, W. E., Kruisbeek, A. M., Wagner, G., and Reinherz, E. L. (2007). Importance of the CD3 $\gamma$ ectodomain terminal $\beta$-strand and membrane proximal stalk in thymic development and receptor assembly. J. Immunol. 178, 3668-3679.

van der Merwe, P. A., and Dushek, O. (2011). Mechanisms for T cell receptor triggering. Nat. Rev. Immunol. $11,47-55$.

Wang, J., Lim, K., Smolyar, A., Teng, M.K., Liu, J.-H., Tse, A. G. T., Liu, J., Hussey, R. E., Chishti, Y., Thomson, C. T., Sweet, R. M., Nathenson, S. G., Chang, H. C., Sacchettini, J. C., and Reinherz, E. L.. (1998). Atomic structure of an $\alpha \beta \mathrm{T}$ cell receptor (TCR) heterodimer in complex with an anti-TCR Fab fragment derived from a mitogenic antibody. EMBO J. $17,10-26$.

Wang, Y., Becker, D., Vass, T., White, J., Marrack, P., and Kappler, J. W. (2009). A conserved CXXC motif in CD3epsilon is critical for $T$ cell development and TCR signaling. PLoS Biol. 7, e1000253. doi:10.1371/journal.pone.0018079

Werlen, G., Hausmann, B., and Palmer, E. (2000). A motif in the alphabeta T-cell receptor controls positive selection by modulating ERK activity. Nature 406, 422-426.

White, J., Haskins, K. M., Marrack, P., and Kappler, J. (1983). Use of I region-restricted, antigen-specific $\mathrm{T}$ cell hybridomas to produce idiotypically specific anti-receptor antibodies. J. Immunol. 130, 1033-1037.
Xu, C., Call, M. E., and Wucherpfennig, K.W. (2006). A membrane-proximal tetracysteine motif contributes to assembly of $\mathrm{CD} 3 \delta \epsilon$ and $\mathrm{CD} 3 \gamma \epsilon$ dimers with the $\mathrm{T}$ cell receptor. $J$. Biol. Chem. 281, 36977-36984.

Xu, C., Gagnon, E., Call, M. E., Schnell, J. R., Schwieters, C. D., Carman, C. V., Chou, J. J., and Wucherpfennig, K. W. (2008). Regulation of T cell receptor activation by dynamic membrane binding of the $\mathrm{CD} 3$ epsilon cytoplasmic tyrosine-based motif. Cell 135, 702-713.

Yanagi, Y., Yoshikai, Y., Leggett, K., Clark, S. P., Aleksander, I., and Mak, T. W. (1984). A human T cell-specific cDNA clone encodes a protein having extensive homology to immunoglobulin chains. Nature 308, 145-149.

Yokosuka, T., Kobayashi, W., SakataSogawa, K., Takamatsu, M., Hashimoto-Tane, A., Dustin, M. L., Tokunaga, M., and Saito, T. (2008). Spatiotemporal regulation of $\mathrm{T}$ cell costimulation by TCR-CD28 microclusters and protein kinase $\mathrm{C}$ theta translocation. Immunity 29, 589-601.

Zech, T., Ejsing, C. S., Gaus, K., de Wet, B., Shevchenko, A., Simons, K., and Harder, T. (2009). Accumulation of raft lipids in T-cell plasma membrane domains engaged in TCR signalling. EMBO J. 28 , 466-476.

Conflict of Interest Statement: The authors declare that the research was conducted in the absence of any commercial or financial relationships that could be construed as a potential conflict of interest.

Received: 27 January 2012; accepted: 27 March 2012; published online: 18 April 2012.

Citation: Kim ST, Shin Y, Brazin $K$, Mallis RJ, Sun Z-YJ, Wagner $G$, Lang MJ and Reinherz EL (2012) TCR mechanobiology: torques and tunable structures linked to early $T$ cell signaling. Front. Immun. 3:76. doi: 10.3389/fimmu.2012.00076

This article was submitted to Frontiers in $T$ Cell Biology, a specialty of Frontiers in Immunology.

Copyright (c) 2012 Kim, Shin, Brazin, Mallis, Sun, Wagner, Lang and Reinherz. This is an open-access article distributed under the terms of the Creative Commons Attribution Non Commercial License, which permits noncommercial use, distribution, and reproduction in other forums, provided the original authors and source are credited. 